\title{
Terrain Recognition Improves the Performance of Neural-Machine Interface for Locomotion Mode Recognition
}

\author{
Ding Wang, Lin Du, He Huang, Senior Member, IEEE
}

\begin{abstract}
Neural-machine interface (NMI) for artificial limbs is a typical biomedical CPS that requires seamless integration of cyber components with physical systems (i.e. prostheses and users). In this paper we aimed to adopt a bio-inspired concept to improve the performance of a NMI for artificial legs by introducing additional information about the walking environment ahead of the prosthesis user. First, a terrain recognition module based on a portable laser distance sensor and an inertial measurement unit (IMU) was designed to accurately classify the terrain type in front of the prosthesis user. The output of this module was then modeled as prior probability and integrated into a Bayesian-based NMI system. The cyber algorithms were real-time implemented and evaluated on an able-bodied subject wearing a passive prosthetic leg in the laboratory environment. The preliminary results showed that the terrain recognition module can accurately recognize the type of terrain in front of the user, approximately half to one second before the critical timing for prosthesis control mode change. NMI with or without the terrain recognition module accurately predicted all the tested task mode transitions. However, the NMI with the terrain recognition module yielded approximately $5 \%$ higher classification accuracy rate in static state and $30 \sim 105 \mathrm{~ms}$ earlier prediction of mode transitions than the NMI without prior knowledge of environmental information. The preliminary results demonstrated the soundness of the bio-inspired concept and established CPS framework to further enhance the accuracy and response time of NMI for artificial leg control.
\end{abstract}

\section{INTRODUCTION}

Cyber-physical system (CPS) is an integrated engineering system, in which a computing and communication core is designed to monitor, coordinate, or control one or more physical systems. The rapid advance in CPS foundation and technology has made it possible to advance biomedical tools, wired/wireless communication, monitor and control of electric power grid, and more.

Neural-machine interface (NMI) is a typical example of biomedical CPS. NMI integrates human and machine (physical systems) via advance cyber components such as communication, neural decoding algorithms, and powerful computing hardware. In the current NMI applications, neural signals collected from brain, peripheral nerves, or muscles from the user have been used to identify the intended movement direction or joint motion[1-3]; the NMI output has been majorly applied to control the movement direction of a computer cursor or a wheel chair, or to operate the joint motion of upper limb prostheses or exoskeleton. The

D Wang, L Du, H Huang are with the Department of Electrical, Computer, and Biomedical Engineering, University of Rhode Island, Kingston, RI, 02881, USA (dwang@ele.uri.edu, 1du@ele.uri.edu, huang@ele.uri.edu).

This work was partly supported by NSF\#1149385, NSF\#0931820, NIH\#RHD064968A, and DOD \#W81XWH-09-2-0020. application of NMI to neural control of lower limb prostheses or exoskeleton has been, however, very limited because of (1) the lack of powered lower limb devices as the control platform and (2) the difficulty in deciphering non-stationary neural signals for stable control of multi-body dynamics.

Recent development of powered prosthetic knee and ankle [4-7] has renewed interest in the idea of neural control of artificial legs [4, 8-11]. In our group, we developed a novel phase-dependent pattern classification strategy to deal with non-stationary electromyographic (EMG) signals for accurate recognition of locomotion mode (e.g. level-ground walking, stair ascent, stepping over obstacles, etc.)[10]. This algorithm was later improved by fusing EMG signals with the mechanical signals measured from artificial legs together [12]. Despite the promising performance of our NMI, false decisions about locomotion mode transitions were sometimes observed. In addition, unstable NMI decisions occasionally occurred during locomotion mode transitions, which led to delayed prediction of mode transition. These errors impeded the application of our developed NMI for reliable prosthetic leg control.

One possible solution to further enhance the performance of our NMI for artificial legs was to introduce additional information about walking environment into the CPS to formulate an environment-aware NMI. This concept was inspired by the biological systems of human locomotion control mechanism, in which human use visual information about the environment to guide their walking pattern. Our previous study [13] explored this concept. Information about the walking terrain was simulated and integrated into the classifiers in NMI as the prior probability. The results showed that the developed cyber algorithm seamlessly integrated three physical systems, including the prosthesis, its user, and the environment, for accurate locomotion mode recognition. However, this previous study was based on offline analysis of simulated prior knowledge of walking environment. To demonstrate the potential of this biomedical CPS, a terrain recognition system should be built and implemented in NMI. Laser-based sensors have been widely used for different terrain recognition systems in mobile robot, autonomous car navigation, and aircraft orientation system, but they are too big or too heavy to be implemented into our NMI. So in this paper, our study aimed (1) to design a wearable terrain recognition method to obtain realistic information of walking terrain and (2) to real-time implement and preliminarily evaluate this environment-aware NMI. The study results can inform the future design of an environment-aware NMI for powered lower limb prosthetics and orthotics. 


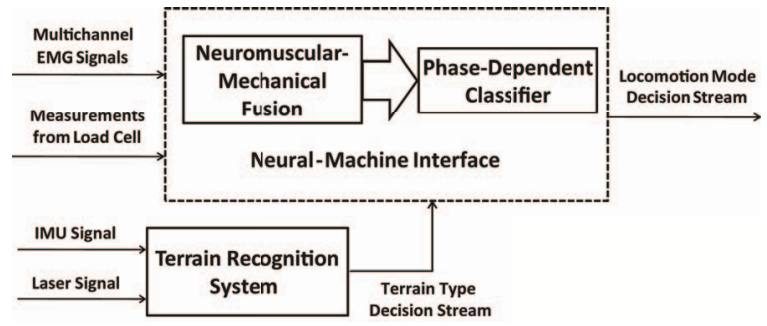

Fig. 1. Architecture of proposed environment-aware NMI for powered artificial legs.

\section{METHODS}

\section{A. Architecture of Designed CPS}

Our proposed CPS consists of two subsystems: a NMI and a terrain recognition system (Fig. 1). The inputs of NMI were the electromyographic (EMG) signals recorded from the residual limb of leg amputees and ground reaction forces and moments on the prosthetic pylon. Neuromuscular-mechanical fusion and phase-dependent pattern classification strategy were used in the NMI to identify the user's locomotion mode, such as level-ground walking and stair ascent $[10,12,14]$. A terrain recognition system recognizes the walking terrain in front of the user based on the inputs from a laser distance sensor and an inertia measurement unit (IMU). The information of walking environment is designed to be obtained before the modulation of the user's muscle activation pattern and biomechanical pattern while walking; such information can be used to reinforce the decision-making in the NMI.

\section{B. Integrating Information of Walking Environment to Fusion-based Neural-Machine Interface}

The approach for integrating information of walking terrain in front of the user with the NMI based on neuromuscular-mechanical fusion was reported in our previous study[13]. First, in NMI, linear discriminant analysis (LDA) was used to accurately classify the user's locomotion mode. The discriminant function of LDA was

$$
d_{c_{i}}=\bar{f}^{T} \Sigma^{-1} \overline{\mu_{l}}-\frac{1}{2} \bar{\mu}_{\imath}^{T} \Sigma^{-1} \overline{\mu_{l}}+\ln P\left(C_{i}\right)
$$

Where, $C_{i}$ is the index for each class, $\bar{f}$ is the observed testing feature vector, $\Sigma$ is the covariance of the training features for all classes, $\overline{\mu_{l}}$ is the mean feature vector for class $i$ in the training set, and $P\left(C_{i}\right)$ denotes the prior probability. Next, the information of walking terrain was modeled as prior probability $P\left(C_{i}\right)$ based on the principle of maximum entropy. For example, if the upcoming terrain is detected as up ramp, the prior probability of ramp ascent will increase while other prior probabilities will decrease. So the LDA decision is more likely to be ramp ascent than other locomotion. The related details can be found in our previous publication [13].

\section{Terrain Recognition Module Design}

A terrain recognition system was designed to classify the walking terrain in front of the user. This system consisted of three modules: stand/turn detection, terrain reconstruction, and terrain classification (Fig. 2).

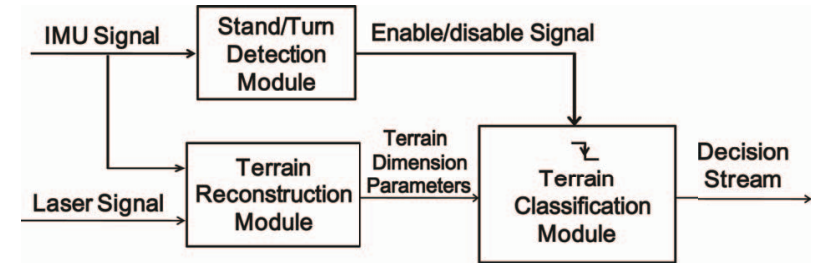

Fig. 2. The block diagram of terrain recognition subsystem

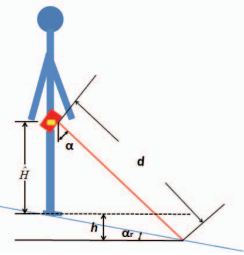

(a) standing on down ramp

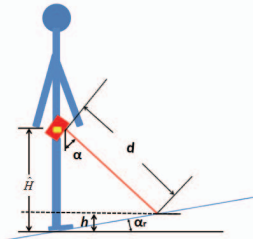

(b) standing on up ramp Fig. 3. Sensor configuration in the terrain recognition system. (a) and (b) show two situations considered to determine the thresholds $T_{\text {low }}$ and

$T_{u p}$ in a decision-tree-based terrain classification algorithm.

In the terrain reconstruction module, a laser sensor and an IMU placed on the waist of a user (Fig. 3) were used to reconstruct the terrain ahead of a user in the sagittal plane when he/she was walking (Fig. 3). The initial angle of the laser beam ( $\alpha$ in Fig. 3 ) in the standing position was set to 45 degrees. Ambulation of the user was necessary to allow the laser to scan the terrain and reconstruct its sagittal profile. In addition, when the user was turning, the scanned terrain did not align with the user's ambulation path. Hence, the function of IMU sensor was not only to measure the orientation of laser beam ( $\alpha$ in Fig. 3) for terrain reconstruction, but also to detect the movement status of users in stand/turn detection module. When the user was in a standing position or made turns, the terrain classification module was disabled (Fig. 2). When the user was walking, based on the reconstructed terrain dimension, the terrain classification module identified the terrain type ahead.

Detailed design of three modules was as follows:

Stand/turn detection module: The IMU measurements (3-axial acceleration and angular velocity) were sampled at $100 \mathrm{~Hz}$. A Kalman filter was used to reduce the noise in the IMU signals. To detect standing, we first computed the signal-magnitude area $(S M A)$ as

$S M A[n]=\sum_{i=0}^{N-1}(|x[n-i]|+|y[n-i]|+|z[n-i]|)$

Wherein, $x[n], y[n]$, and $z[n]$ indicated the acceleration along the $\mathrm{x}, \mathrm{y}$, and $\mathrm{z}$-axes of the IMU for the sample $n$, respectively. $N$ was set as 50 . When $S M A$ was smaller than a threshold (calibrated as the maximum SMA value in the initial standing position), the status of standing was detected. The detection of turns was achieved by monitoring the angle displacement in horizontal plane, which was calculated based on the angular velocity of IMU. A turn was detected when the rotation angle was greater than 30 degrees.

Terrain reconstruction module: Based on the signals of two sensors (sampled at $100 \mathrm{~Hz}$ ), two parameters of the terrain dimension were reconstructed. The first parameter was the terrain height $h[n]$ from the level of the ground, on which the user currently walked on (Fig. 3). It was calculated by

$$
h[n]=\widehat{H}-d[n] \cos (\alpha[n])
$$


$d[n]$ is the distance between the laser sensor and the terrain scanned by the laser beam (Fig. 3), measured by the laser sensor; $\alpha[n]$ is the angle between the laser beam and the vertical direction, obtained by the IMU (Fig. 3); $\widehat{H}$ denotes the height of the laser sensor measured when the subject was in standing. A thirty-point moving average filter was used to smooth $h[n]$. The second parameter $s[n]$ was defined as

$$
s[n]=\left\{\begin{array}{cc}
0 & T_{\text {low }}<h[n] \leq T_{u p} \\
\max \{|\mathrm{h}[\mathrm{n}]|, \mathrm{s}[\mathrm{n}-1]\} & \text { otherwise }
\end{array}\right.
$$

$T_{\text {low }}$ and $T_{u p}$ are constant, serving as thresholds for recognizing level ground (refer to next section for details). The parameter $s[n]$ denoted the maximum change of terrain height $h[n]$ when an uneven terrain was identified. These two parameters were then fed to the terrain classification module for identifying the terrain type.

Terrain classification module: A decision tree was designed to classify the terrain type ahead of the user (Fig. 4). In the first node of the decision tree, the terrain height $h$ was used as the feature to classify the terrains that were above (upper terrain in Fig. 4), below (lower terrain in Fig. 4), and equal (level terrain in Fig. 4) the current level of walking terrain. To calculate the threshold applied to $h$, assuming that a user stands on a down ramp (Fig. 3a) and up ramp (Fig. 3b). The terrain height in these two situations was denoted as $h_{\text {low }}$ by (5) and $h_{\text {up }}$ by (6), respectively.

$$
\begin{aligned}
h_{\text {low }} & =-\frac{\widehat{H}}{\cot (\alpha) \cot \left(\alpha_{r)}-1\right.} \\
h_{u p} & =\frac{\widehat{H}}{\cot (\alpha) \cot \left(\alpha_{r}\right)+1}
\end{aligned}
$$

Wherein, $\alpha_{r}$ is the slope of the ramp. Since a reconstructed height of a staircase is larger than $h$ of ramp, in order to accurately classify uneven terrains, the thresholds $T_{\text {low }}$ and $T_{\text {up }}$ should be no larger than $h_{\text {low }}$ and $h_{u p}$. In this study, we defined $T_{\text {low }}=0.8 \times h_{\text {low }}$ and $T_{u p}=0.8 \times h_{u p}$.

In the second level of the decision tree, the nodes of lower terrain and upper terrain were further separated into down/up ramp, down/up stair, and "others" based on the second parameters $[n]$. The class of "others" includes the objects, such as tables and wall, which were not interested terrains for the NMI design. When the user walked on the stairs, $s[n]$ value was significantly larger than that when the user walked on inclines. Therefore, the thresholds to separate the stair and ramp were defined as $T_{\text {upstair }}=T_{\text {downstair }}=$ stair $\_$; where, stair $h$ is the height of a staircase. In addition, if $s[n]$ was greater than $T_{\text {others } 1}=T_{\text {others } 2}=3 \times$ stair_ $h$, it was very likely that the laser scanned some object other than our interested terrain, and the decision of the terrain recognition

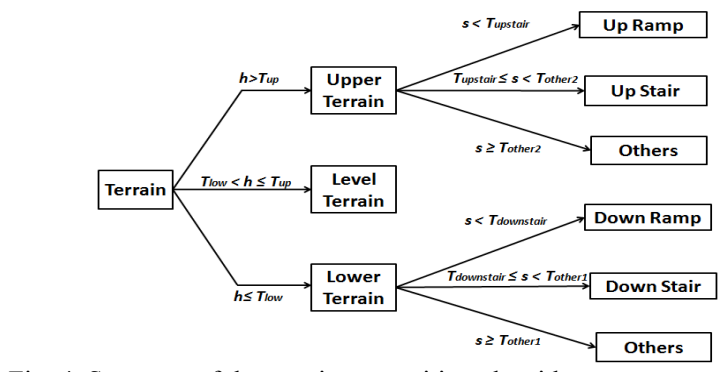

Fig. 4. Structure of the terrain recognition algorithm. system was "others". The factor 3 was selected because the laser beam can usually scan one or two staircases ahead of the user when walking on the stairs. Finally, a 30-point majority vote was applied to the output of terrain recognition system to remove some decision errors. It was noteworthy that the parameters of terrain recognition system depended only on the defined dimension of the terrains, e.g. height of a staircase; they were the same for all the human subjects as long as the value of $\widehat{H}$ was calibrated. Hence, this system was easy to set up.

\section{Real-Time Implementation}

The environment-aware NMI were implemented on a PC (2.93 GHz 2 Duo CPU and 3 GB RAM) using MATLAB in real-time. Based on the U.S. building codes, the ramp slope ranges within 5-12 degrees, and the stair riser heights are limited between $102 \mathrm{~mm}$ and $209 \mathrm{~mm}$. Therefore, $\alpha_{r}=$ 5 degrees and stair_h=102 $\mathrm{mm}$, which were used to compute the thresholds in decision tree. The output of the terrain recognition subsystem was connected to the NMI. When the recognized terrain was level terrain, up/down ramp, or up/down stairs, the prior probability in the discriminant function of NMI as (1) was modulated accordingly based on the maximum entropy principle [13]. Otherwise, the output of terrain recognition system did not adjust the boundaries of classifiers in the NMI. To evaluate the real-time environment-aware NMI, one able-bodied subject (AB01) was recruited with his consent and the Institutional Review Board (IRB) approval.

(1) Evaluation of Terrain Recognition: A portable optical laser distance sensor (Leuze Electronic, MI, US) was instrumented on the right waist of the subject. A commercially available IMU (Xsens, Netherland) was attached to the laser sensor. The intersection angle between the laser beam and $\mathrm{x}$-direction axis of the IMU was fixed at 45 degrees. In order to evaluate the system performance, two extra IMU sensors were put on both feet of the subject to monitor the gait events. The signals from the laser sensor and the IMU sensors were sampled at $100 \mathrm{~Hz}$ and all the data recordings in this study were synchronized.

An obstacle course was built in the laboratory, consisting of a level-ground walkway, 5-step stairs (the height of each stair is $160 \mathrm{~mm}$ ), and 10 -feet ramp (the slope is 8 degree). We also put chairs and tables in the testing area to introduce noise. The experiment consisted of two sessions: calibration session and real-time testing session. During calibration, the subject was instructed to stand still on the level ground for 20 seconds; the initial $\alpha$ value was around 45 degrees. These baseline data were used to derive $\widehat{H}$ and $S M A$ in standing for standing detection.

In real-time testing, the subject walked at a comfortable speed and started each trial from standing still. In each trial, the subject was asked to define his own walking path along the obstacle course. In addition, we encouraged the subject to purposely walk towards walls and along the office furniture. Totally 10 trials were conducted. 
(2) Evaluation of Environment-aware NMI: During this experiment, AB01 wore a prosthetic leg using a plastic adaptor. Seven channels of surface EMG signals from the thigh muscles were monitored by an EMG system (Motion Lab System, US). Mechanical forces and moments were measured by a six-degree-of-freedom load cell (Bertec Corporation, OH, US). The setup of the laser distance sensor and IMU were the same as described above. The EMG and mechanical signals were both sampled at $1000 \mathrm{~Hz}$. All the input data were synchronized.

Six locomotion modes including level-ground walking (W), stair ascent (SA), stair descent (SD), ramp ascent (RA), ramp descent (RD) and standing (ST) were investigated. The experiment contained two sessions: training session and testing session. In the training session, training data for each class was collected in an individual trial and used to estimate the parameters of the classifiers in the NMI. In the testing trial, the subject initiated level-ground walking from a standing position, randomly chose to his walking path along the obstacle course. Twenty trials were conducted in total.

\section{E. System Evaluation Parameters}

(1) Evaluation of Terrain Recognition: Our previous study defined critical timings for NMI to identify the transitions between the user's locomotion modes [12]. For all the transitions from level ground walking, the critical timing was the beginning of swing phase to other terrain. For all the transitions to level ground walking, the critical timing was the beginning of weight acceptance on the level ground. The two IMU sensors placed on the instep of each foot were used to find these gait events [15]. If the upcoming terrain cannot be correctly recognized before the defined critical timing, a missed terrain transition was identified. The number of missed terrain transitions was used to evaluate the system accuracy. If the upcoming terrain change was correctly recognized before the critical timing, the time elapse between recognition of terrain change to the critical timing was used to evaluate the time response of the terrain recognition system.

(2) Evaluation of Environment-aware NMI: Three evaluation parameters as we reported previously [12-13] were used to quantify the performance of NMI: (1) overall accuracy rate for classifying the user's locomotion mode in static state, (2) the number of missed transitions between the user's locomotion modes, and (3) the mode transition prediction time. The same critical timing as mentioned above was used to determine the latter two parameters in mode transitions [12-13]. Offline post-processing on the data was conducted to evaluate the system performance without the terrain recognition system.

\section{RESUlts}

\section{A. Terrain Recognition Results}

There were total 80 terrain transitions in the testing trials of $\mathrm{AB} 01$. All the transitions were correctly detected before the defined critical timing. The response time for recognizing terrain transitions in Table 1 shows that all the terrain transitions could be recognized half to one second before the critical timing. The terrain recognition result of
TABLE I. PREDICTION TIME OF TERRAIN TRANSITIONS (UNITS: MS)

\begin{tabular}{ccccccccc}
\hline & LG- & UR- & LG- & DR- & LG- & US- & LG- & DS- \\
& UR & LG & DR & LG & US & LG & DS & LG \\
\hline \multirow{3}{*}{ AB01 } & 630 & 672 & 584 & 490 & 554 & 700 & 1204 & 720 \\
& \pm & \pm & \pm & \pm & \pm & \pm & \pm & \pm \\
& 136 & 345 & 201 & 236 & 401 & 93 & 262 & 264 \\
\hline
\end{tabular}

Note: level ground (LG), up stair (US), down stair (DS), up ramp (UR), down ramp (DR)

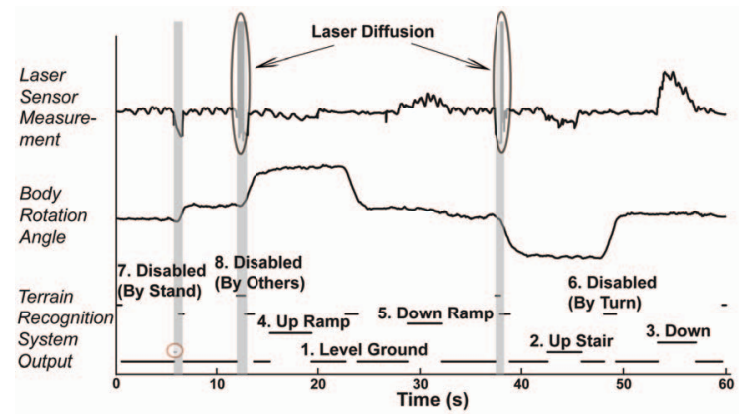

Fig. 5. Terrain recognition results in one representative trial.

one representative trial of $\mathrm{AB} 01$ was demonstrated in Fig. 5. Laser sensor measurement changed when it hit different terrains. The gray areas indicated that the laser hit the objects (i.e. others) other than normal terrains. Sometimes the laser beam diffused, and the measurement became very large. In this case, the output of terrain recognition system was "others". Body rotation angle indicated whether the user was walking straight or making a turn. There was one error in the representative trial, highlighted by the red circle. When the laser first hit the wall, the decision of the system was "up stair" first and then changed to "others" after $170 \mathrm{~ms}$.

\section{B. Environment-aware NMI Result}

The overall classification accuracy rate for six locomotion modes across 20 real-time testing trials was $95.48 \%$ with the terrain recognition system. The offline analysis showed the

TABLE II PREDICTION TIME OF MODE TRANSITIONS (UNIT: MS)

\begin{tabular}{ccccccccc}
\hline \multirow{2}{*}{ Transition } & W- & SA- & W- & SD- & W- & RA- & W- & RD- \\
& SA & W & SD & W & RA & W & RD & W \\
\hline \multirow{2}{*}{ W/o Terrain } & 220 & 345 & 185 & 265 & 335 & 220 & 455 & 325 \\
recognition System & \pm & \pm & \pm & \pm & \pm & \pm & \pm & \pm \\
& 111 & 96 & 94 & 142 & 108 & 106 & 123 & 193 \\
\hline \multirow{2}{*}{ W/ Terrain } & 250 & 410 & 220 & 335 & 415 & 255 & 560 & 400 \\
recognition System & \pm & \pm & \pm & \pm & \pm & \pm & \pm & \pm \\
& 105 & 110 & 108 & 155 & 138 & 117 & 120 & 183 \\
\hline
\end{tabular}

Note: W, SA, SD, RA and RD denote level-ground walking, stair ascent, stair descent, ramp ascent and ramp descent respectively.

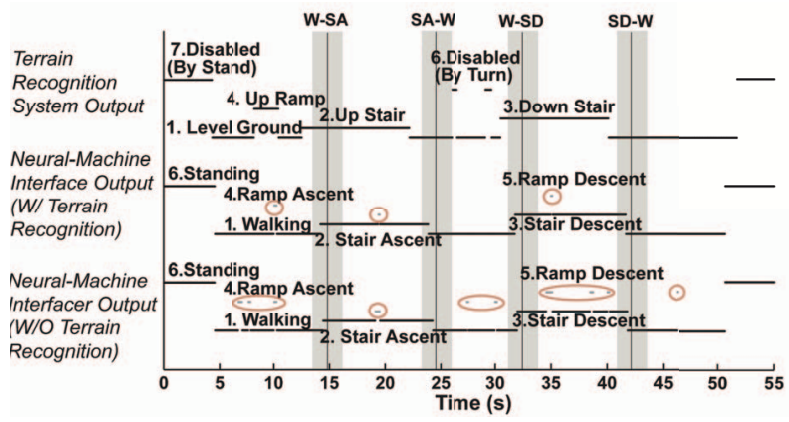

Fig. 6. Real-time decision of terrain recognition system, environment-aware NMI, and NMI without terrain recognition system in one trial. The gray area represents the locomotion mode transitional period. The gray vertical line indicates the critical timing for each transition. NMI errors were also highlighted by red circles. 
classification accuracy would decrease to $90.64 \%$ without the assistance of terrain recognition. For all 20 trials, all the mode transitions were predicted before the defined critical timing in the real-time testing. The prediction time for 8 types of mode transitions with and without the terrain recognition system is shown in Table 2. The NMI could detect the mode transitions 30 105 ms earlier with the terrain recognition system. The real-time decisions of the terrain recognition system and NMI systems in one trial are depicted in Fig. 6. It was noteworthy that the laser scanned an up ramp within the first 10 seconds of the trial; however, AB01 did not step on the ramp. Interestingly, in the meantime, the decisions of the NMI with terrain recognition module were correct, except one error. It indicated that the information about walking environment did not dominate the decision in NMI for user intent recognition.

\section{DISCUSSION}

This study demonstrated preliminary development of a biomedical CPS that used real-time cyber algorithms to integrate human, prosthesis, and environment (physical systems) together for neural control of artificial legs.

Our designed wearable terrain recognition system based on a portable laser sensor and IMU was simple and effective. The system parameters were easy to set up and consistent for the users. The preliminary result showed that the designed system could accurately recognize the terrain change in front of the user approximately half to one second before the critical timing. Most of the errors in the terrain recognition system occurred when the laser hit the walls or furniture in the environment. When the laser swept the bottom part of chairs, tables, or wall, the system misclassified them as up terrains initially and then quickly switched back to the correct decision.

Real-time implementation of environment-aware NMI proved the soundness of our design concept and CPS engineering framework. The terrain recognition module significantly improved the performance of NMI for user intent recognition: it eliminated many NMI errors and yielded an earlier prediction of locomotion mode transition. In addition, the information about the terrain ahead of the user only assisted rather than dominated the NMI decision. This was because the NMI still provided correct decisions about the user's locomotion mode when errors in the terrain recognition system occurred or when the laser scanned a terrain but the user did not decide to step on that terrain. Therefore, our designed environment-aware NMI was robust to the noisy or incorrect terrain recognition decisions.

In spite of these promising results, there were several study limitations. First, we only presented the preliminary results derived from one able-bodied subject. Additionally, many parameters in terrain recognition system, such as the configuration of the laser sensor, needed to be investigated and optimized for maximize the system performance and practice. It is our future work to further optimize the proposed CPS and evaluate it on lower limb amputees wearing powered prostheses.

\section{CONCLUSION}

In this paper, a real-time environment-aware NMI was designed for locomotion mode recognition for artificial legs. The designed wearable terrain recognition system can accurately recognize the type of terrain in front of the user in a noisy environment. The obtained environmental information reinforced the decision-making process in the NMI and, therefore, improved the accuracy and response time for locomotion mode recognition. Moreover, this study also showed that the environment-aware NMI was robust to the errors in the terrain recognition system. These results showed the potential application of designed CPS to neural control of powered prosthetic legs. The developed cyber algorithms and real-time implementation may benefit other biomedical CPS design with human-in-the-loop.

\section{REFERENCES}

[1] P. Zhou, M. M. Lowery, K. B. Englehart, H. Huang, Li G., Hargrove L., J. P. A. Dewald, and K. Kuiken, "Decoding a New Neural-Machine Interface for Control of Artificial Limbs," $J$ Neurophysiol, 2007.

[2] T. A. Kuiken, L. A. Miller, R. D. Lipschutz, B. Lock, K. A Stubblefield, P. Marasco, P. Zhou, and G. A. Dumanian, "Targeted Reinnervation for Enhanced Prosthetic Arm Function in Woman with a Proximal Amputation," Lancet, vol. 369, pp. 371-380, 2007.

[3] H. Huang, P. Zhou, G. Li, and T. A. Kuiken, "An analysis of EMG electrode configuration for targeted muscle reinnervation based neural machine interface," IEEE Trans Neural Syst Rehabil Eng, vol. 16, pp. 37-45, 2008.

[4] S. Au, M. Berniker, and H. Herr, "Powered ankle-foot prosthesis to assist level-ground and stair-descent gaits," Neural Netw, vol. 21, pp. 654-66, 2008.

[5] E. C. Martinez-Villalpando and H. Herr, "Agonist-antagonist active knee prosthesis: a preliminary study in level-ground walking," $J$ Rehabil Res Dev, vol. 46, pp. 361-73, 2009.

[6] F. Sup, A. Bohara, and M. Goldfarb, "Design and Control of a Powered Transfemoral Prosthesis," International Journal of Robotics Research, vol. 27, pp. 263-73, 2008.

[7] M. Liu, P. Datseris, and H. Huang, "A prototype for smart prosthetic legs: analysis and mechanical design," in International Conference on Control, Robotics and Cybernetics. New Delhi, India: IEEE, 2011, pp. $139-43$.

[8] K. H. Ha, H. A. Varol, and M. Goldfarb, "Volitional control of a prosthetic knee using surface electromyography," IEEE Trans Biomed Eng, vol. 58, pp. 144-51, 2011.

[9] L. J. Hargrove, A. M. Simon, R. D. Lipschutz, S. B. Finucane, and T A. Kuiken, "Real-time myoelectric control of knee and ankle motions for transfemoral amputees," JAMA, vol. 305, pp. 1542-4, 2011.

[10] H. Huang, T. A. Kuiken, and R. D. Lipschutz, "A strategy for identifying locomotion modes using surface electromyography," IEEE Trans Biomed Eng, vol. 56, pp. 65-73, 2009.

[11] F. Zhang, S. E. D'Andrea, M. J. Nunnery, S. M. Kay, and H. Huang, "Towards design of a stumble detection system for artificial legs," IEEE Trans Neural Syst Rehabil Eng, vol. 19, pp. 567-77, 2011.

[12] H. Huang, F. Zhang, L. J. Hargrove, Z. Dou, D. R. Rogers, and K. B. Englehart, "Continuous locomotion-mode identification for prosthetic legs based on neuromuscular-mechanical fusion," IEEE Trans Biomed Eng, vol. 58, pp. 2867-75, 2011.

[13] L. Du, F. Zhang, M. Liu, and H. Huang, "Towards Design of an Environment-aware Adaptive Locomotion-Mode-Recognition System " IEEE Trans Biomed Eng, vol. 59, pp. 2716-25, 2012.

[14] X. R. Zhang, Y. H. Liu, F. Zhang, J. Ren, Y. L. Sun, Q. Yang, and H. Huang, "On Design and Implementation of Neural-Machine Interface for Artificial Legs," IEEE Transactions on Industrial Informatics, vol. 8, pp. 418-429, 2012.

[15] A. M. Sabatini, C. Martelloni, S. Scapellato, and F. Cavallo, "Assessment of walking features from foot inertial sensing," IEEE Trans Biomed Eng, vol. 52, pp. 486-94, 2005. 